\title{
DECOMPOSITION THEOREMS OF RIEMANNIAN MANIFOLDS
}

\author{
BY
}

\section{PYNG WANG}

\begin{abstract}
Given two complementary orthogonal parallel foliations on a complete connected Riemannian manifold $M$, a necessary and sufficient condition for the direct product of the two leaves through a point $m$ being a covering manifold of $M$ is obtained. It is shown that the direct product of the two leaves through $m$ of the two foliations is a Riemannian covering of $M$ if the two leaves are regular at $m$. Moreover, if one of the foliations is regular and the intersection of the two leaves through $m$ contains only the point $m$, then $M$ is is ometric to the direct product of the two leaves.
\end{abstract}

1. Introduction. Let $M$ be a connected complete Riemannian manifold and let $\Phi$ be the holonomy group of $M$ at $m \in M$. Suppose that $\Phi$ preserves a proper subspace $T_{m}^{1}$ of the tangent space $T M_{m}$. If $M$ is simply connected, then de Rham's decomposition theorem asserts that $M$ is isometric to the direct product of the maximal integral manifolds of the distributions $T^{1}$ and $T^{2}$ obtained by parallel translation of $T_{m}^{1}$ and $T_{m}^{2}=\left(T_{m}^{1}\right)^{\perp}$ over $M$. In this paper we want to extend de Rham's decomposition theorem to a Riemannian manifold which is not assumed to be simply connected. First, we give a necessary and sufficient condition for the direct product of the two maximal integral manifolds through $m$ to be a covering manifold of $M$ (Theorem 1). Then we use Theorem 1 to prove that the direct product of the maximal integral manifolds tbrough $m$ is a Riemannian covering manifold of $M$ if the two maximal integral manifolds are regular at $m$ (Theorem 2). This involves a study of the relation between the fundamental groups of the maximal integral manifolds and the fundamental group of $M$.

Assume further that the foliation induced by one of the distribution is regular and the intersection of the two maximal integral manifolds through $m$ contains only the point $m$. We use Theorem 2 to prove that $M$ is isometric to the direct product of the two maximal integral manifolds through $m$ (Theorem 3 ).

The author sincerely thanks Professor $\mathrm{Ph}$. Tondeur for his direction and encouragement. The results in this paper will serve as partial fulfillment of the thesis requirement for the author's Ph.D. candidacy at the University of Illinois in Urbana-Champaign.

Received by the editors July 6, 1972.

AMS (MOS) subject classifications (1970). Primary 53C20, 53C65; Secondary 58A30, $53 \mathrm{C} 35$. 
2. Statement of Theorem 1. Let $M$ be a connected Riemannian manifold. Suppose $T M_{m}$, the tangent space of $M$ at a fixed point $m$, is decomposed into two orthogonal subspaces $T_{m}^{1}$ and $T_{m}^{2}$ which are invariant under the action of the holonomy group $\Phi$ of $M\left(\right.$ at $m$ ). Then parallel translating $T_{m}^{i}$ to every point of $M$ gives distributions $T^{i}$ which are involutive and hence integrable. Let $\mathfrak{F}_{i}$ be the foliations induced by $T^{i}$ and $M_{i}$ the leaves of $\mathfrak{F}_{i}$ through $m, i=1,2$. Denote $q_{i}: \tilde{M}_{i} \rightarrow M_{i}$ the universal covering space of $M_{i}, i=1,2$ and $q: \tilde{M} \rightarrow M$ the universal covering space of $M$.

Theorem 1. There is an isometry $\Psi: \tilde{M}_{1} \times \tilde{M}_{2} \rightarrow \tilde{M}_{\text {. The covering projection }}$ $p=q \circ \Psi: \tilde{M}_{1} \times \tilde{M}_{2} \rightarrow M$ has the following property $p \mid \tilde{M}_{i}=q_{i}, i=1,2$. A neces. sary and sufficient condition for $p$ to factorize through $q_{1} \times q_{2}$ and a Riemannian covering map $\lambda: M_{1} \times M_{2} \rightarrow M$ is the following condition:

(A) the parallel translation along any closed curve at $m$ in $M_{i}$ leaves the vectors orthogonal to $M_{i}$ invariant, $i=1,2$.

Corollary 1. If the holonomy group of $M$ at $m$ is trivial, then $M_{1} \times M_{2}$ is a Riemannian covering space of $M$. In particular, $\pi_{1}\left(M_{1}\right) \times \pi_{1}\left(M_{2}\right), \pi_{1}\left(M_{1}\right)$ and $\pi_{1}\left(M_{2}\right)$ are subgroups of $\pi_{1}(M)$.

Corollary 2. If $M_{i}$ are simply connected $i=1,2$, then $M_{1} \times M_{2}$ is a Riemannian covering space of $M$.

3. Preliminaries. Let $q: \tilde{M} \rightarrow M$ be a Riemannian covering and $\tilde{\gamma}(t), 0 \leq t$ $\leq 1$ a curve in $\tilde{M}$. Denote $\gamma(t)=q \circ \tilde{\gamma}(t)$ in $M$. Let $\tau_{\tilde{\gamma}}$ and $\tau_{y}$ be the parallel translations along $\tilde{\gamma}$ in $\tilde{M}$ from $\tilde{\gamma}(0)$ to $\tilde{\gamma}(1)$ and along $\gamma$ in $M$ from $\gamma(0)$ to $\gamma(1)$ respectively. Then we have

Lemma 1. $q_{*} \tilde{\gamma} \tilde{X}=r_{\gamma} q_{*} \tilde{X}$ for $\tilde{X} \in T \tilde{M}_{\mathcal{Y}(0)}$.

Proof. Locally, $q: \tilde{M} \rightarrow M$ is an isometry. Then choose a finite number of neighborhoods covering $\tilde{\gamma}(t)$. This proves the lemma.

Let $T$ be an involutive distribution on a manifold $M$ with $\operatorname{dim} M=m$. A cubic coordinate system $\left(x^{1}, \ldots, x^{m}, U\right)$ is called flat with respect to $T$ if, for each $y \in U,\left(\partial / \partial x^{1}\right)_{y}, \cdots,\left(\partial / \partial x^{n}\right)_{y}$ is a base for $T_{y}$.

We say that $M$ bas a local product structure of type $\left(m_{1}, m_{2}\right)$ if there exists a direct sum decomposition $T M=T^{1} \oplus T^{2}$ of the tangent bundle of $M$ into smooth subbundles of dimension $m_{i}>0$ such that both tangent fields $T^{i}$ are completely integrable. We will write $\mathfrak{F}_{i}$ for the foliation induced by $T^{i}$.

Let $M$ be a smooth m-manifold with a local product structure $\left(\mathfrak{F}_{1}, \mathfrak{F}_{2}\right)$ of type $(k, m-k)$. Then for each point $x \in M$ there exists a cubic coordinate system $\left(x^{1}, \ldots, x^{m}, U\right)$ centered at $x$ which is flat with respect to $\mathfrak{F}_{1}$ and $\mathfrak{F}_{2}$. 
That is, the slices $x^{k+1}=c_{k+1}, \cdots, x^{m}=c_{m}$ and the slices $x^{1}=c_{1}, \cdots, x^{k}=c_{k}$ are the integral manifolds of $\mathfrak{F}_{1}$ and $\mathfrak{F}_{2}$ respectively.

Denote $U_{1}$ the set of points in $U$ with coordinate $\left(x^{1}, \ldots, x^{k}, 0,0, \ldots, 0\right)$, $U_{2}$ the set of points in $U$ with coordinate $\left(0,0, \ldots, 0, x^{k+1}, \ldots, x^{m}\right)$. Then $U_{i}$ are the coordinate neighborhoods of $x$ in $M_{i}(x)$, the leaves of $\mathfrak{F}_{i}$ through $x$. Consider the map $f_{U}: U_{1} \times U_{2} \rightarrow U$ such that $f_{U}\left(\left(x^{1}, \ldots, x^{k}\right),\left(x^{k+1}, \ldots, x^{m}\right)\right)=$ $\left(x^{1}, \cdots, x^{m}\right)$. We have the following criterion for foliations to be parallel.

Proposition 1. Let $M$ be a connected Riemannian manifold with metric $g$. Assume that $M$ has a local product structure $\left(\mathfrak{F}_{1}, \mathfrak{F}_{2}\right)$ which is orthogonal. Then the following statements are equivalent.

(i) For each point $x \in M$ there exists a flat coordinate system $U$ centered at $x$ such that the corresponding diffeomorphism $f_{U}: U_{1} \times U_{2} \rightarrow U$ is an isometry.

(ii) The associated tangent fields $T^{i}$ are parallel and, for all $x \in M, \phi \in \Phi_{x}$, $\phi T_{x}^{i}=T_{x}^{i}, i=1,2$.

(iii) For each $x \in M$, the leaves $M_{i}(x), i=1,2$, are totally geodesic.

Proof. (ii) $\Rightarrow$ (i), (ii) $\Rightarrow$ (iii) and (iii) $\Rightarrow$ (ii) are standard [3]. We want to prove $(\mathrm{i}) \Rightarrow$ (ii). Let $S^{1}$ and $S^{2}$ be the tangent fields associated to the foliations with the two slices $U_{1} \times\left\{y_{2}\right\}$ and $\left\{y_{1}\right\} \times U_{2}$ as leaves in $U_{1} \times U_{2}$, where $y_{i} \epsilon$ $U_{i}$. Then $S^{i}, i=1,2$ are parallel with respect to the product metric in $U_{1} \times U_{2}$. Since $f_{U}$ maps the slices in $U_{1} \times U_{2}$ onto the slices in $U$ which are the integral manifolds of some $T^{i}$, it follows that $\left(f_{U}\right)_{*} S^{i}=T^{i}, i=1,2$. Hence $T^{i}$ are parallel in $U$. Since any given curve can be covered by a finite number of flat neighborhoods with corresponding isometries $f_{U}$, we have proved that $T^{i}$ are parallel in $M$.

Let $F(x)$ be the leaf through $x$ of a foliation $₹$. If for $y \in F(x)$ there exists a flat neighborhood $U$ of $y$ such that each leaf of $₹$ intersects $U$ in only one slice, then the leaf is called regular at $y$. The leaf $F(x)$ is said to be regular if it is regular at every point of $F(x)$. And $₹$ is a regular foliation if each leaf is regular.

4. Statement of Theorem 2 and examples.

Theorem 2. Suppose that the leaves through $m, M_{i}=M_{i}(m)$, are regular at $m$, $i=1,2$. Then $M_{1} \times M_{2}$ is a Riemannian covering space of $M$. In particular, $\pi_{1}\left(M_{1}\right) \times \pi_{1}\left(M_{2}\right), \pi_{1}\left(M_{1}\right)$ and $\pi_{1}\left(M_{2}\right)$ are subgroups of $\pi_{1}(M)$.

We say that a local product structure $\left(\mathfrak{F}_{1}, \mathfrak{F}_{2}\right)$ on a Riemannian manifold $M$ is compatible with the metric if the $\mathfrak{F}_{i}$ are orthogonal, $i=1,2$ and $\left(\mathfrak{F}_{1}, \mathfrak{F}_{2}\right)$ satisfies one of the conditions in Proposition 1. Using this definition we can restate Theorem 1 and Theorem 2.

Theorem 2'. Let $M$ be a connected complete Riemannian manifold witb a 
local product structure $\left(\mathfrak{F}_{1}, \mathfrak{F}_{2}\right)$ which is compatible with the metric. Suppose that there exists a point $x \in M$ such that the leaves $M_{i}=M_{i}(x)$ of $\mathfrak{F}_{i}$ through $x$ are regular at $x$ or such that $M_{i}$ satisfy condition $(A)$ at $x$. Then $\lambda: M_{1} \times M_{2}$ $\rightarrow M$ is a Riemannian covering. In particular, $\pi_{1}\left(M_{1}\right) \times \pi_{1}\left(M_{2}\right), \pi_{1}\left(M_{1}\right)$ and $\pi_{1}\left(M_{2}\right)$ are subgroups of $\pi_{1}(M)$.

Corollary 3. Let $M$ be a connected complete Riemannian manifold with a compatible local product structure $\left(\mathfrak{F}_{1}, \mathfrak{F}_{2}\right)$. Suppose that the leaves $M_{i}=M_{i}(x)$ are regular at $x$. Assume further that the covering map $\lambda$ induces a surjective map and bence an isomorphism between the fundamental groups of $M_{1} \times M_{2}$ and the fundamental group of $M$. Then $M$ is isometric to $M_{1} \times M_{2}$.

Corollary 4. Let $M$ be a connected complete Riemannian manifold with a compatible local product structure $\left(\mathfrak{F}_{1}, \mathfrak{F}_{2}\right)$. Suppose that $\pi_{1}(M)$ bas no nontrivial subgroup isomorpbic to $\pi_{1}\left(M_{1}(x)\right)$. Then the leaf $M_{1}(x)$ is simply connected.

Example 1. Consider $T^{2}$ the flat torus. Let $\mho_{1}$ be the foliation in $T^{2}$ with leaves projected by the parallel lines with fixed irrational slope in $\mathbf{R}^{2}$, with $\mathfrak{F}_{2}$ the perpendicular foliation. Then $\left(\mathfrak{F}_{1}, \mathfrak{F}_{2}\right)$ is a local product structure compatible with the metric. For $x \in T^{2}$, the leaves $M_{i}(x)$ equal to $R, i=1,2$. We have that $M_{1}(x) \times M_{2}(x)=\mathbf{R} \times \mathbf{R}$ is a covering space of $T^{2}$. The covering map is obtained from the standard covering map by rotating through this fixed irrational angle. Both foliations $\mho_{1}$ and $\mho_{2}$ are not regular. In fact, the leaves $M_{i}(x)$ are not regular at any point. Since the holonomy group of the flat torus $T^{2}$ is trivial, $M_{i}(x)$ satisfy condition $(A)$ at $x$.

Example $2\left(s^{2} \times \mathbf{R}\right)$. I owe special thanks to Professor F. Kamber who made some corrections on this example. Let $Z_{2}$ act on $S^{2} \times \mathbf{R}$ by $a(x, y)=(-x,-y)$, where $a$ is the nontrivial element in $Z_{2}$, and $p: S^{2} \times \mathbf{R} \rightarrow M=S^{2} \times \mathbf{R} / \mathbf{Z}_{2} \cdot M$ has an obvious local product structure compatible with the metric induced from $S^{2} \times \mathbf{R}$ and $\pi_{1}(M)=Z_{2}$. Denote $(\overline{x, y})=p(x, y) \in M$. At the points $(\overline{x, y}), x \in$ $s^{2}, 0 \neq y \in \mathbf{R}$. We have $M_{1}((\overline{x, y})) \approx S^{2}, M_{2}((\overline{x, y})) \approx \mathbf{R}$ and both leaves are simply connected. We see that $M_{1}((\overline{x, y})) \times M_{2}((\overline{x, y})) \approx S^{2} \times \mathbf{R}$ is a covering of $M$. At the points $(\overline{x, 0})$, we have $M_{1}((\overline{x, 0})) \approx \mathbf{P}_{2}$ and $M_{2}((\overline{x, 0})) \approx \mathbf{R}$. We claim that $M_{1}((\overline{x, 0})) \times M_{2}((\overline{x, 0})) \approx \mathbf{P}_{2} \times \mathbf{R}$ is not a covering space of $M$. First noting that $M$ is the canonical line bundle of $P_{2}$ and $\pi_{1}(M)=Z_{2}$ has no nontrivial subgroup, we observe that if this were the case, the covering map $\lambda$ from $\mathbf{P}_{2} \times \mathbf{R}$ to $M$ would induce a surjective map of fundamental groups which would imply $M \approx$ $\mathbf{P}_{2} \times \mathbf{R}$. Then $\lambda$ induced from $S^{2} \times \mathbf{R}$ would map $\mathbf{P}_{2} \times 0$ to the 0 -section of the canonical line bundle $M$ and we would have $M-\{0$-section $\} \approx \mathbf{P}_{2} \times \mathbf{R}-\mathbf{P}_{2} \times 0$ which is disconnected with two components. This would imply that the canonical line bundle is trivial. We see that the leaf $\left.M_{1}(\overline{x, 0})\right)=\mathbf{P}_{2}$ is not regular at 
$(\overline{x, 0})$ and does not satisfy the condition $(A)$ at $(\overline{x, 0})$. For instance, take the only nontrivial homotopic closed curve $\gamma$ at $(\overline{x, 0})$ in $\mathbf{P}_{2}$. Then ${ }^{r}{ }_{y} X=-X$ for $X$ orthogonal to $M_{1}((\overline{x, 0}))$ at $(\overline{x, 0})$. This example shows that the condition $(\Lambda)$ in Theorem 1 cannot be removed.

5. Proof of Theorem 1. First, we will show that $p: \tilde{M}_{1} \times \tilde{M}_{2} \rightarrow M$ is a Riemannian covering where $\tilde{M}_{i}$ is the universal covering manifold of $M_{i}$ (Lemma 2). Then we factorize the map $p$ through $M_{1} \times M_{2}$ as follows:

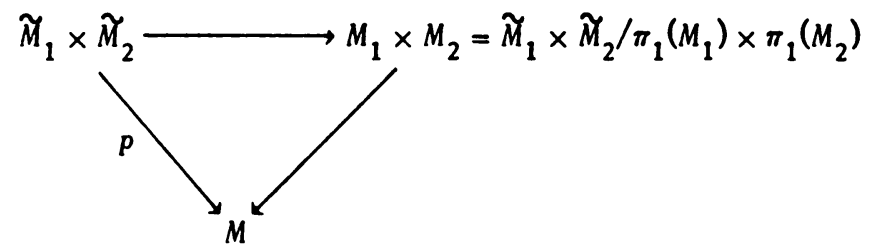

For this we have to prove that $\pi_{1}\left(M_{1}\right) \times \pi_{1}\left(M_{2}\right)$ is a subgroup of $\pi_{1}(M)$ (Lemmas $3,4,5,6)$.

Lemma 2. Let $q: \tilde{M} \rightarrow M$ and $q_{i}: \tilde{M}_{i} \rightarrow M$ be the universal covering manifolds of $M$ and $M_{i}$ respectively. There is an isometry $\Psi: \tilde{M}_{1} \times \tilde{M}_{2} \rightarrow \tilde{M}^{\circ}$. The covering projection $p=q \circ \Psi: \tilde{M}_{1} \times \tilde{M}_{2} \rightarrow \tilde{M}$ bas the following property $p \mid \tilde{M}_{i}=$ $q_{i}, i=1,2$.

Proof. Let $\tilde{m} \in q^{-1}(m)$. We have $T \tilde{M}_{\tilde{m}} \oplus \tilde{T}_{\tilde{m}}^{2}$ is the lifting of $T_{m}^{i}$ at $\tilde{m}$. By Lemma $1, \tilde{T}_{\tilde{m}}^{i}, i=1,2$, are invariant under the action of $\underset{\sim}{\tilde{\Phi}}$, the holonomy group of $\tilde{M}$ at $\widetilde{m}_{\text {. }}$. Parallel translating $\widetilde{T}_{\widetilde{m}}^{i}$ to every point in $\tilde{M}$ gives involutive distributions $\tilde{T}^{i}, i=1,2$ which are the same as the distributions obtained by lifting the distributions $T^{i}, i=1,2$ according to Lemma 1 .

Let $M_{i}^{\prime}$ be the maximal integral manifolds of $\tilde{T}^{i}$ through $\tilde{m}$ in $\tilde{M}, i=1,2$. Applying de Rham's decomposition theorem we have $\tilde{M} \approx M_{1}^{\prime} \times M_{2}^{\prime}$, where $M_{i}^{\prime}$ are simply connected complete Riemannian manifolds. It remains to show that $M_{i}^{\prime} \approx$ $\tilde{M}_{i}$. Consider the map $q \mid M_{i}^{\prime}$. Since $M_{i}^{\prime}$ is the maximal integral manifold to $\widetilde{T}^{i}$ through $\tilde{m}$ and $\tilde{T}^{i}$ is the lifting of $T^{i}$, we have $q \mid M_{i}^{0}$ mapping $M_{i}^{\prime}$ into $M_{i}$. Using the uniqueness of lifting of a curve at $\tilde{m}$ and its tangent vectors at $\tilde{m}$, we see that $q \mid M_{i}^{\prime}: M_{i}^{\prime} \rightarrow M_{i}$ is onto. Moreover, it is a local isometry. Hence $q \mid M_{i}^{\prime}: M_{i}^{\prime}$ $\rightarrow M_{i}$ is a covering space $\left[3\right.$, p. 176]. Therefore $\tilde{M}_{\tilde{N}} \approx \tilde{M}_{1} \times \tilde{M}_{2}$. With this identification, it is clear that $p \mid \tilde{M}_{i}=q_{i}$.

Lemma 3. Let $\tilde{M}$ be a simply connected, connected Riemannian manifold and $\underset{\sim}{\Gamma}$ a discrete group of isometries of $\tilde{M}$ with fixed point free action. Denote $M=\tilde{M} / \Gamma$ and $p: \tilde{M} \rightarrow M$ the projection. Let $T$ be an involutive $C^{\infty}$-distribution on $M$ which is invariant under the action of the restricted bolonomy group of $M$ at a fixed point $m$. Denote by $S_{\tilde{m}}$ the lifting of $T_{m}$ at $\tilde{m} \in p^{-1}(m), S$ the 
distribution in $\tilde{M}$ obtained by parallel translating $S_{\tilde{m}}$ to every point of $\tilde{M}$. Then $T_{m}$ is invariant under the action of the bolonomy group of $M$ at $m$ if and only if $\phi_{*} S_{\tilde{\boldsymbol{x}}}=S_{\phi(\tilde{x})}$ for all $\phi \in \Gamma, \tilde{x} \in \tilde{M}$.

Proof. Let $\tilde{a}(t)$ and $\tilde{\gamma}(t)$ be curves joining $\tilde{m}, \tilde{x}$ and joining $\tilde{x}, \phi(\tilde{x})$ respectively. Denote $\alpha(t)=p \tilde{\alpha}(t), \gamma(t)=p \tilde{\gamma}(t)$. By definition of the distribution $S$, we have $S_{\widetilde{x}}=\tau_{\widetilde{\alpha}} S_{\widetilde{m}}$. Now

$$
\begin{aligned}
& \phi_{*} S_{\tilde{x}}=\text { lifting of } p_{*} S_{\tilde{x}} \quad \text { at } \phi(\tilde{x}), \\
& =\text { lifting of } p_{*}^{\tau} \tilde{a}^{S_{m}} \text { at } \phi(\tilde{x}), \\
& =\text { lifting of } \tau_{a} p_{*} S_{\tilde{m}} \text { at } \phi(\tilde{x}) \text { (Lemma 1), } \\
& =\text { lifting of } \tau_{a} T_{m} \quad \text { at } \phi(\tilde{x}) \text {; } \\
& S_{\phi(\tilde{x})}=\tau \tilde{y}^{\tau} \tilde{a}^{\prime} S_{m} \\
& =\text { lifting of } \tau_{\gamma} \tau_{\alpha} p_{*} S_{\tilde{m}} \text { at } \phi(\tilde{x}), \\
& =\text { lifting of } \tau_{\gamma} \gamma_{a} T_{m} \text { at } \phi(\tilde{x}) \text {. }
\end{aligned}
$$

If $T_{m}$ is invariant under the action of the holonomy group of $M$ at $m$, then

$$
\tau_{a-1}{ }^{\tau} \gamma^{\tau} a_{m} T_{m}=T_{m} \text { or }{ }^{\tau} \gamma_{a}^{\tau} T_{m}=\tau_{a} T_{m}
$$

Hence $\phi_{*} S_{\tilde{x}}=S_{\phi(\tilde{x})}$ for all $\phi \in \Gamma$ and $\tilde{x} \in \tilde{M}$. Conversely, choose $\tilde{x}=\tilde{m}$ and apply Lemma 1.

Lemma 4. Let $\Gamma$ be the group of deck transformations of the covering $p: \tilde{M}_{1} \times \tilde{M}_{2} \rightarrow M$ and $I\left(\tilde{M}_{i}\right)$ the isometry groups of $\tilde{M}_{i}, i=1,2$. Consider $I\left(\tilde{M}_{i}\right) \times$ $I\left(\tilde{M}_{2}\right)$ as a subgroup of $I\left(\tilde{M}_{1} \times \tilde{M}_{2}\right)$. Then we bave $\Gamma \subset I\left(\tilde{M}_{1}\right) \times I\left(\tilde{M}_{2}\right)$.

Proof. It suffices to show that for $\phi \in \Gamma$ there exist isometries $\phi_{i}: \tilde{M}_{i} \rightarrow$ $\tilde{M}_{i}, i=1,2$, such that $\underset{\sim}{\phi}(x)=\left(\phi_{1}\left(x_{1}\right), \phi_{2}\left(x_{2}\right)\right)$ for $x=\left(x_{1}, x_{2}\right) \in \tilde{M}_{1} \times \widetilde{M}_{2}$. We prove that, if $p_{i}: \widetilde{M}_{1} \times \widetilde{M}_{2} \rightarrow \widetilde{M}_{i}$ denotes the natural projection, then $p_{i}(\phi(x))$ depends only on $x_{i}=p_{i}(x)$. Given any point $y=\left(x_{1}, y_{2}\right)$, let $\gamma_{2}(t)$ be a curve in $\widetilde{M}_{2}$ such that $\gamma_{2}(0)=x_{2}, \gamma_{2}(1)=y_{2}$. Then the curve $\gamma(t)$ defined by $\left(x_{1}, \gamma_{2}(t)\right)$ has the tangent vectors $\dot{\gamma}(t)$ belonging to the distribution $\tilde{T}^{2}$. By Lemma 3 , $\phi_{*}(\dot{\gamma}(t))$ belongs to the same distribution for all $t$. Hence $p_{1}(\phi(\gamma(t)))$ is independent of $t$. In particular, $p_{1}(\phi(x))=p_{1}(\phi(y))$. Then we define $\phi_{1}\left(x_{1}\right)=p_{1}(\phi(x))$. Similarly, we can define $\phi_{2}\left(x_{2}\right)=p_{2}(\phi(x))$. We have $\phi(x)=\left(\phi_{1}\left(x_{1}\right), \phi_{2}\left(x_{2}\right)\right)$ and $\phi_{i} \in I\left(\tilde{M}_{i}\right)$. 
Lemma 5. Let $\lambda_{i}: M_{i} \rightarrow M$ be the inclusion map. Then the homomorphism $\left(\lambda_{i}\right)_{*}: \pi_{1}\left(M_{i}\right) \rightarrow \pi_{1}(M)$ is injective.

Proof. Let $\rho_{i}: \tilde{M}_{i} \rightarrow \tilde{M}_{i} \times \tilde{M}_{2}$ be the injection. Using the fact that $p \mid \tilde{M}_{i}$ maps $\tilde{M}_{i}$ into $M_{i}$, we have the following commutative diagram:

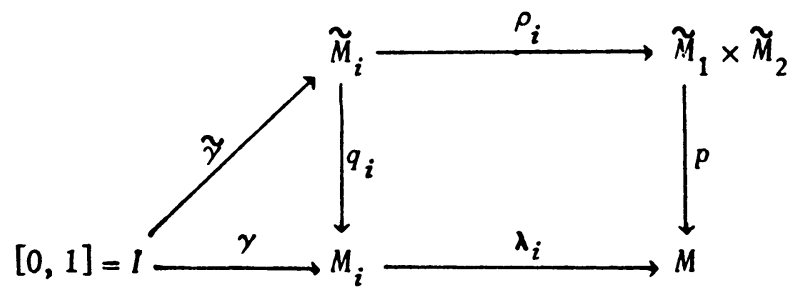

where $\tilde{\gamma}$ is the lifting of $\gamma$ at $\tilde{m}$.

If $\lambda_{i} \circ \gamma \sim 0$ (homotopic to the constant loop at $m$ ), we want to prove $\gamma \sim 0$. From the diagram, $\rho_{i} \circ \tilde{\gamma}$ is the lifting of $\lambda_{i} \circ \gamma$ at $(\tilde{m}, \tilde{m})$. Since the lifting of $\lambda_{i} \circ \gamma$ is a loop, we have $\rho_{i} \circ \tilde{\gamma}(0)=\rho_{i} \circ \tilde{\gamma}(1)$. But the fact that $\rho_{i}$ is an injection implies that $\tilde{\gamma}$ is a loop. That is, $\gamma \sim 0$.

Lemma 6. Consider $\left(\lambda_{1}\right)_{*} \pi_{1}\left(M_{1}\right)$ as a subgroup of deck transformations of the covering $p: \tilde{M}_{1} \times \tilde{M}_{2} \rightarrow M$. Then $\left(\lambda_{1}\right)_{*} \pi_{1}\left(M_{1}\right) \subset I\left(\tilde{M}_{1}\right) \times$ Id if and only if the parallel translation ${ }^{r} y$ along any closed curve $y$ at $m$ in $M_{1}(m)$ leaves vectors in $T_{m}^{2}$ invariant.

Proof. Let $\Gamma_{\gamma}$ be the deck transformation in $\tilde{M}_{1} \times \tilde{M}_{2}$ induced by $\gamma$ in $M_{1}(m)$. Since $p \mid \tilde{M}_{1}(\tilde{m})$ maps $\tilde{M}_{1}(\tilde{m})$ onto $M_{1}(m)$, the lifting $\tilde{\gamma}$ of $\gamma$ at $\tilde{m}$ lies in $\tilde{M}_{1}(\tilde{m})$. We have $\Gamma_{\gamma} \underset{\sim}{(\tilde{m})}=\tilde{\gamma}(1)$. Let $\tilde{x}$ be a point in $\tilde{M}_{2}(\tilde{m}), \tilde{a}$ the geodesic joining $\tilde{m}$ and $\tilde{x}$ in $\tilde{M}_{2}(\tilde{m})$. Denote $a=p \circ \tilde{a}$. Assume that $r_{y} X=X$ for $X \epsilon$ $T_{m}^{2}$. Since

$$
\begin{aligned}
p_{*}{ }^{\tau} \tilde{\gamma} \dot{\tilde{\alpha}}(0) & =\tau_{\gamma} p_{*} \dot{\tilde{\alpha}}(0) \\
& =\tau_{\gamma} \dot{\alpha}(0) \\
& =\dot{\alpha}(0),
\end{aligned}
$$

${ }{ }_{\tilde{\gamma}} \dot{\tilde{a}}(0)$ is the lifting of $\dot{\alpha}(0)$ at $\tilde{\gamma}(1)=\Gamma_{\gamma}(\tilde{m})$. Hence the lifting of $a$ at $\tilde{\gamma}(1)$ is a geodesic $\beta$ starting at $\tilde{\gamma}(1)$ with initial vector $\tau \tilde{\tilde{a}}(0)$. By the product structure of $\tilde{M}_{1} \times \tilde{M}_{2}, \beta$ is the parallel translation of $\tilde{\alpha}$. Hence $\beta(1)=\Gamma_{\gamma}(\tilde{x})$ lies in $\tilde{M}_{1}(\tilde{x})$. Let $\tilde{y}$ be an arbitrary point in $\tilde{M}_{1}(\tilde{m}) \times \tilde{M}_{2}(\tilde{m})$. By the product structure of $\tilde{M}_{1}(\tilde{m}) \times \tilde{M}_{2}(\tilde{m})$, there exists a point $\tilde{x}$ in $\tilde{M}_{2}(\tilde{m})$ such that $\tilde{x}$ is the intersection of $\tilde{M}_{1}(\tilde{y})$ and $\tilde{M}_{2}(\tilde{m})$. Let $\tilde{\delta}$ be a curve joining $\tilde{x}, \tilde{y}$ in $\tilde{M}_{1}(\tilde{x})=\tilde{M}_{1}(\tilde{y})$ and $\delta=p \circ \tilde{\delta}$ in $M_{1}(x)$. Using the fact that $p \mid \tilde{M}_{1}(\tilde{x})$ maps $\tilde{M}_{1}(\tilde{x})$ onto $M_{1}(x)$ and 
$\Gamma_{\gamma}(\tilde{x})$ lies in $\tilde{M}_{1}(\tilde{x})$, we prove that the lifting of $\delta$ starting at $\Gamma_{\gamma}(\tilde{x})$ lies in $\tilde{M}_{1}(\tilde{x})$. Hence $\Gamma_{\gamma}$ is a deck transformation leaving the second coordinate fixed. That is, $\left(\lambda_{1}\right)_{*} \pi_{1}\left(M_{1}\right) \subset I\left(\tilde{M}_{1}\right) \times I d$.

Conversely, the fact that $\left(\lambda_{1}\right)_{*} \pi_{1}\left(M_{1}\right) \subset I\left(\tilde{M}_{1}\right) \times$ Id implies $\Gamma y$ leaving the second coordinate fixed. By the unique lifting property, $\beta$ is the lifting of $a$ at $\Gamma_{\gamma}(\tilde{m})$. Hence $p_{*} \dot{\beta}(0)=\dot{\alpha}(0)$. Since $\beta(0)=\tau_{\tilde{y}} \tilde{\tilde{a}}(0)$, we have $\dot{\alpha}(0)=p_{*}{ }^{\tau} \dot{\gamma}^{\dot{\alpha}}(0)=$ $r_{\gamma} p_{*} \dot{\tilde{a}}(0)=\tau_{\gamma} \dot{\alpha}(0)$. End of the proof of Theorem 1 .

From Lemma 5 we may consider $\pi_{\sim}\left(M_{i}\right)$ as a subgroup of $\pi_{1}(M)$, hence as a group of deck transformations in $\tilde{M}_{1} \times \widetilde{M}_{2}$. By Lemma 6 , we see that $\left(\lambda_{1}\right)_{*} \pi_{1}\left(M_{1}\right) C$ $I\left(\tilde{M}_{1}\right) \times$ Id and $\left.\lambda_{2}\right)_{*} \pi_{1}\left(M_{2}\right) \subset$ Id $\times I\left(\tilde{M}_{2}\right)$. Together with Lemma 4 , we have

$$
\begin{aligned}
& \Gamma \approx \pi_{1}(M) \subset \quad l\left(\tilde{M}_{1}\right) \times l\left(\tilde{M}_{2}\right) \\
& u \quad u \\
& \left(\lambda_{1}\right)_{*} \pi_{1}\left(M_{1}\right) \quad\left(\lambda_{2}\right)_{*} \pi_{1}\left(M_{2}\right)
\end{aligned}
$$

Hence $\pi_{1}\left(M_{1}\right)$ commutes with $\pi_{1}\left(M_{2}\right)$, both considered as subgroups of $\pi_{1}(M)$. Therefore the map $\lambda_{*}: \pi_{1}\left(M_{1}\right) \times \pi_{1}\left(M_{2}\right) \rightarrow \pi_{1}(M)$ defined by

$$
\lambda_{*}\left(\phi_{1}, \phi_{2}\right)=\left(\lambda_{1}\right)_{*} \phi_{1} \cdot\left(\lambda_{2}\right)_{*} \phi_{2}
$$

is a homomorphism.

From the fact $\left(\lambda_{1}\right)_{*} \pi_{1}\left(M_{1}\right) \cap\left(\lambda_{2}\right)_{*} \pi_{1}\left(M_{2}\right)=$ Id and the injectivity of $\left(\lambda_{i}\right)_{*}$ it follows that $\lambda_{*}$ is injective.

Conversely, if $p$ can be factorized through $q_{1} \times q_{2}$ and a Riemannian covering map $\lambda: M_{1} \times M_{2} \rightarrow M$, then $\left(\lambda_{1}\right)_{*} \pi_{1}\left(M_{1}\right)$ considered as a subgroup of $\pi_{1}(M)$ lies in $I\left(\tilde{M}_{1}\right) \times$ Id. Now Lemma 6 implies that the condition is necessary. This completes the proof of the theorem.

\section{Proof of Theorem 2.}

Lemma 7. If the leaf $M_{1}(m)$ is regular at $m$ then ${ }^{\gamma} X=X$ for $X \in T_{m}^{2}$ and $\gamma$ a closed curve in $M_{1}(m)$.

Proof. Let $U$ be a flat coordinate neighborhood of $\mathrm{m}$ which is convex and simple. Choose a number $t_{1}$ such that $\gamma\left[0, t_{1}\right] \subset U$. Denote ${ }^{\top} \gamma\left(t_{1}\right) X$ the parallel translation of $X$ to $\gamma\left(t_{1}\right)$ along $\gamma$. Let $\exp s X$ be the endpoint of the geodesic with initial unit vector $X$ in $T_{m}^{2}$ and arc length $s$. In view of the local product structure, there exists a small number $s$ such that $\exp s X$ and $\exp s\left(\tau_{\gamma\left(\iota_{1}\right)} X\right)$ lie in the same leaf. Choosing a finite number of this kind of neighborhoods cove ering $\gamma(t)$, we conclude that $\exp s X$ and $\exp s\left(r_{\gamma(1)} X\right)=\exp s\left(r_{y} X\right)$ lie in the same leaf if $s$ is sufficiently small. If we choose the neighborhood $U$ in which $M_{1}(m)$ is regular at $m$, then $\exp s X$ and $\exp s\left(r_{\gamma} X\right)$ are in the same slice. Since 
$\exp s X$ and $\exp s\left(\tau_{\gamma} X\right)$ are in $U_{2}$, they are in the intersection of the fixed slice and $U_{2}$. Hence $\exp s X=\exp s(\tau, X)$. Therefore $X=\tau_{y} X$ by the uniqueness of geodesics in the neighborhood $U$.

Now Theorem 2 follows from Theorem 1 and Lemma 7.

7. Global decomposition. The following characterization of a regular foliation is well known.

Lemma 8. Let $\mathfrak{F}$ be a foliation in a manifold $M$, then the set of leaves $M / \mathfrak{F}$ bas a unique differentiable structure sucb that $\eta: M \rightarrow M / \mathcal{F}$ is a differentiable map if and only if $\mathfrak{F}$ is regular [4].

For a local product structure this can be strengthened as follows.

Proposition 2. Let $M$ be a connected complete Riemannian manifold with a local product structure $\left(\mathfrak{F}_{1}, \mathfrak{F}_{2}\right)$ which is compatible with the Riemannian metric. Suppose that $\mathfrak{F}_{1}$ is a regular foliation. Then $\eta: M \rightarrow B$ is a fibre bundle with the base manifold $B=M / \mho_{1}$, the set of leaves of $\mho_{1}$. Moreover, $B$ is a complete Riemannian manifold (Hausdorff). $B$ is covered by $M_{2}(x)$, the leaf of $F_{2}$ through $x$ if $M_{2}(x)$ is regular or satisfies condition $(A)$ at $x$.

Proof. The compatible local product structure implies the metric is "bundlelike". By a theorem of Reinhart [7], we have only to prove the last statement. Denote $M_{i}=M_{i}(x)$, the leaves of $\mho_{i}, i=1,2$. Let $\mathcal{S}_{1}$ and $\mathcal{S}_{2}$ be the two natural foliations in $M_{1} \times M_{2}$ with leaves $M_{1} \times\left\{y_{2}\right\}$ and $\left\{y_{1}\right\} \times M_{2}$ respectively. Denote $S^{i}$ the tangent fields associated to $\delta_{i}$.

Since $\lambda: M_{1} \times M_{2} \rightarrow M$ is a Riemannian covering and the tangent fields $T^{i}$ associated to $\mathfrak{F}_{i}$ are parallel, as in the proof of Lemma 2, we have $\lambda_{*} S^{i}=T^{i}$, $i=1,2$. This means that the integral manifolds $M_{1} \times\left\{y_{2}\right\}$ of $S^{1}$ map into the leaves of $T^{1}$, and therefore

$$
\bar{\lambda}: M_{2}=M_{1} \times M_{2} / \mathcal{S}_{1} \rightarrow M / \mathscr{F}_{1}=B .
$$

Since $\mathfrak{F}_{1}$ is regular, it is easy to see that $B$ is a manifold and $\bar{\lambda}$ is a differentiable map. Moreover, the compatibility of the local product structure implies that $B$ is a complete Riemannian manifold and that $\bar{\lambda}$ is a local isometry. Since $M_{2}$ is complete, $\bar{\lambda}$ is a covering map.

Corollary 5. Let $M$ be a compact connected manifold with a local product structure $\left(\mathfrak{F}_{1}, \mathfrak{F}_{2}\right)$. That is, with two complementary transversal foliations $\mathfrak{F}_{1}$ and $\mathfrak{F}_{2}$. Suppose $\mathfrak{F}_{1}$ and $\mathfrak{F}_{2}$ are regular foliations. For each $x \in M$, denote $M_{i}=M_{i}(x)$, the leaves of $\mathfrak{F}_{i}$ through $x, i=1,2$. Then we have

(i) $M_{1} \times M_{2}$ is a covering space of $M$. In particular, $\pi_{1}\left(M_{1} \times M_{2}\right), \pi_{1}\left(M_{1}\right)$ and $\pi_{2}\left(M_{2}\right)$ are subgroups of $\pi_{1}(M)$. 
(ii) $M$ is a fibre bundle over the base manifold $B_{j}=M / F_{i}, i \neq j, i, j=1,2$ and $B_{j}$ is covered by $M_{j}(x)$ for all $x \in M, j=1,2$.

Proof. Since $M$ is compact and $\mathfrak{F}_{i}$ are regular, $B_{j}$ are manifolds (Hausdorff) and $\eta_{j}: M \rightarrow B_{j}$ are differentiable. Choose metrics on $B_{j}, j=1,2$, then pull them back to $M$. We get two metrics $g^{1}$ and $g^{2}$ on $M$. Let $g=g^{1} \oplus g^{2}$. It is easy to see that the local product structure $\left(\mathfrak{F}_{1}, \mathfrak{F}_{2}\right)$ is orthogonal with respect to $g$. We want to prove that it is also compatible with respect to $g$.

Let $y \in M$ and $\left(x^{1}, \ldots, x^{k}, x^{k+1}, \ldots, x^{m}, U\right)$ be a flat coordinate neighborhood with center $y$. Consider the diffeomorphism $f_{U}: U_{1} \times U_{2} \rightarrow U$. By definition of $g$ we see that $f_{U}$ is an isometry at the points $\left(z_{1}, y\right)$ and $\left(y, z_{2}\right)$ for $z_{i} \epsilon$ $U_{i} \subset M_{i}(y)$. To prove $f_{U}$ is an isometry, it remains to show that $g\left(X_{i}, X_{j}\right)$ is independent of $x^{k+1}, \ldots, x^{k}$ and $g\left(X_{a}, X_{\beta}\right)$ is independent of $x^{1}, \ldots, x^{k}$, where $X_{i}$ $=\partial / \partial x^{i}$ and $1 \leq i, j \leq k ; k+1 \leq \alpha, \beta \leq m$. This is also clear by the definition of the pull-back of the metrics. Hence Corollary 5 follows from Proposition 1, Theorem 2 and Proposition 2.

Corollary 6. Let $\mathrm{H}_{1}, \mathrm{H}_{2}$ be two connected closed subgroups of a compact Lie group $G$ such that their Lie algebras are complementary. Then we bave

(i) $H_{1} \times H_{2}$ is a covering space of $G$. In particular, $\pi_{1}(H), \pi_{1}\left(H_{2}\right)$ and $\pi_{1}\left(H_{1} \times H_{2}\right)$ are subgroups of $\pi_{1}(G)$.

(ii) $\pi_{2}\left(G / H_{i}\right)=0, i=1,2$.

Proof. Left translating $H_{1}$ and $H_{2}$ to every point of $G$ gives two regular foliations on G. (i) is clear from Corollary 5. (ii) follows from (i) and the fact that $\pi_{2}(G)=0$, together with the homotopy sequence of the fibrations $H_{i} \rightarrow G \rightarrow$ $G / H_{i}, i=1,2$.

Example 3. That the conclusion (ii) need not hold is seen from the fibration $s^{1} \rightarrow s^{3} \rightarrow s^{2}$.

Lemma 9. Let $M$ be a complete Riemannian manifold with a compatible local product structure $\left(\mathfrak{F}_{1}, \mathfrak{F}_{2}\right)$. Suppose that $\mathfrak{F}_{1}$ is regular and there exists a point $0 \in M$ such that $M_{2}(0)$ is regular at 0 or such that $M_{2}(0)$ satisfies condition (A) at 0 . If $M_{1}(0) \cap M_{2}(0)$ contains only the point 0 , then $M_{1}(x) \cap M_{2}(0)$ also contains only one point for every $x \in M$.

Proof. By definition of $\lambda$ and $\lambda$, we have the following commutative diagram

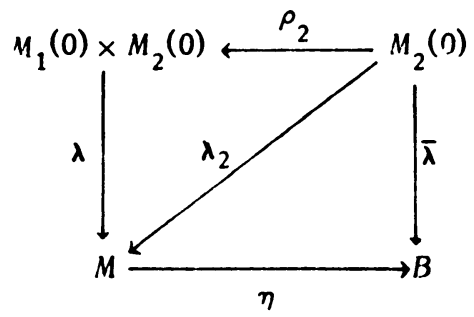


where $\rho_{2}$ is the natural injection and $\lambda_{2}$ the inclusion.

Let $d_{x}=\eta\left(M_{1}(x)\right) \in B$, for $x \in M$. Using the fact $\eta \circ \lambda_{2}=\bar{\lambda}$, we have $\bar{\lambda}^{-1}\left(d_{x}\right)=M_{1}(x) \cap M_{2}(0)$. In particular for $x=0, \bar{\lambda}^{-1}\left(d_{0}\right)=M_{1}(0) \cap M_{2}(0)$ contains only the point 0 . Since $\bar{\lambda}$ is a covering map and $B$ is connected, $M_{1}(x) \cap$ $M_{2}(0)$ contains only one point.

Theorem 3. Let $M$ be a connected complete Riemannian manifold with a lo. cal product structure $\left(\mathfrak{F}_{1}, \mathfrak{F}_{2}\right)$ which is compatible with the metric. Assume that the foliation $\mathfrak{F}_{1}$ is regular and there exists a point $0 \in M$ such that $M_{2}(0)$ is regular at 0 or such that $M_{2}(0)$ satisfies condition $(A)$ at 0 . If $M_{1}(0) \cap M_{2}(0)$ contains only the point 0 , then $M$ is isometric to $M_{1}(0) \times M_{2}(0)$.

Proof. By Lemma 9, we see that $\bar{\lambda}: M_{2}(0) \rightarrow B$ is injective. Hence $\bar{\lambda}$ is a global isometry, i.e. $M_{2}(0) \approx B$. From Proposition $2, \eta: M \rightarrow B$ is a fibre bundle with fibre $M_{1}(0)$. We have the exact homotopy sequence

$$
\rightarrow \pi_{2}(M) \stackrel{\eta_{*}}{\longrightarrow} \pi_{2}(B) \rightarrow \pi_{1}\left(M_{1}(0)\right) \stackrel{\left(\lambda_{1}\right)_{*}}{\longrightarrow} \pi_{1}(M) \stackrel{\eta_{*}}{\longrightarrow} \pi_{1}(B) \rightarrow 0 .
$$

Since $\lambda$ is a diffeomorphism, we identify $B$ with $M_{2}(0)$. Then we have

$$
M \underset{\lambda_{2}}{\stackrel{\eta}{\rightleftarrows}} M_{2}(0) \text { and } \eta_{*}\left(\lambda_{2}\right)_{*}=\text { Id }
$$

Hence we have a short exact sequence

$$
0 \rightarrow \pi_{1}\left(M_{1}(0)\right) \stackrel{\left(\lambda_{1}\right)_{*}}{\longrightarrow} \pi_{1}(M) \stackrel{\eta_{*}}{\stackrel{\left(\lambda_{2}\right)_{*}}{\longrightarrow}} \pi_{1}\left(M_{2}(0)\right) \rightarrow 0 .
$$

Using the splitting at right, we prove that the map $\lambda_{*}: \pi_{1}\left(M_{1}(0)\right) \times \pi_{1}\left(M_{2}(0)\right) \rightarrow$ $\pi_{1}(M)$ defined by $\lambda_{*}(a, \beta)=\left(\lambda_{1}\right)_{*} a \cdot\left(\lambda_{2}\right)_{*} \beta$ is surjective. Hence $M$ is isometric to $M_{1}(0) \times M_{2}(0)$.

Corollary 7. Let $M$ be a connected complete Riemannian manifold with a lo. cal product structure $\left(\mathfrak{F}_{1}, \mathfrak{F}_{2}\right)$ which is compatible with the metric. Suppose that the foliation $\mathfrak{F}_{1}$ is regular and the set of leaves $B=M / \mathfrak{F}_{1}$ is simply connected. If $M_{2}(x)$ is regular at $x$ or satisfies condition $(A)$ at $x$, then $M$ is isometric to $M_{1}(x) \times M_{2}(x)$.

Corollary 8. Let $M$ be a connected compact manifold with two complementary foliations $\mathfrak{F}_{1}$ and $\mathfrak{F}_{2}$. Suppose that the two foliations are regular and either that there exists a point $0 \in M$ such that $M_{1}(0) \cap M_{2}(0)$ contains only the point 0 , or that one of the spaces of leaves is simply connected. Then $M$ is diffeomorpbic to $M_{1}(0) \times M_{2}(0)$. 
8. Applications and examples. Using the arguments and results in Theorem 2 , we have the following two decomposition theorems for symmetric spaces which are not assumed to be simply connected.

Proposition 3. Let $M$ be a connected Riemannian symmetric space. Then $M$ is isometric to a product $N_{1} \times M_{2} \cdot N_{1}$ is a connected Riemannian symmetric space with nonnegative sectional curvature and $M_{2}$ is a simply connected Rie. mannian symmetric space with nonpositive sectional curvature and without Euclidean factors.

Proof. Let $\tilde{M}$ be the universal covering manifold of $M$. Then $\tilde{M} \approx \tilde{M}_{0} \times \tilde{M}_{1}$ $\times \tilde{M}_{2}$, where $\tilde{M}_{0}$ is a Euclidean space, $\tilde{M}_{1}$ and $\tilde{M}_{2}$ are Riemannian symmetric spaces of compact and noncompact type respectively. Let $\tilde{T}_{\sim}^{i}, i=0,1,2$, be the natural foliations on $\tilde{M}$ induced by the product structure of $\tilde{M}$. Since $M$ is a homogeneous manifold, the group of deck transformations $\Gamma$ of the covering $p: \widetilde{M} \rightarrow M$ is a group of Clifford translations $[9, \mathrm{p} .66]$. Using the fact that a Clifford translation preserves the product structure $\left[9\right.$, p. 72], we have $\Gamma \subset I\left(\tilde{M}_{0}\right)$ $\times I\left(\tilde{M}_{1}\right) \times I\left(\tilde{M}_{2}\right)$. That is, if $\phi \in \Gamma$ then $\phi=\left(\phi_{0}, \phi_{1}, \phi_{2}\right)$ where $\phi_{i}: \tilde{M}_{i} \rightarrow \tilde{M}_{i}$, $i=0,1,2$, are Clifford translations. Hence $\widetilde{T}_{i}, i=0,1,2$, induce three mutually orthogonal, parallel distributions $T^{i}$ on $M, i=0,1,2$. Let $0 \in M$ be a reference point and $M_{i}, i=0,1,2$, the leaves of $T^{i}$ through 0 .

Denote $\Gamma_{2}=\left\{\phi_{2} \mid\left(\phi_{0}, \phi_{1}, \phi_{2}\right)=\dot{\varphi} \in \Gamma\right\} . \Gamma_{2}$ is a group of Clifford translations in $\tilde{M}_{2}$ which is a product of irreducible noncompact, non-Euclidean symmetric spaces. By a theorem of Tits $\left[9\right.$, p. 73], we conclude that $\Gamma_{2}=\{$ Id $\}$.

Let $N=\tilde{M}_{0} \times \tilde{M}_{1} \cdot N$ is a Riemannian symmetric space with nonnegative sectional curvature. Then $M \approx N / \Gamma \times \tilde{M}_{2}=N_{1} \times M_{2} \cdot N_{1}$ is a Riemannian symmetric space with nonnegative sectional curvature and $M_{2}$ is a simply connected Riemannian symmetric space with nonpositive sectional curvature and without Euclidean factors.

By Proposition 3, we need only to look at a Riemannian symmetric space with nonnegative sectional curvature. We will impose a condition on the symmetric space to get a decomposition.

Proposition 4. Let $M$ be a connected Riemannian symmetric space with nonnegative sectional curvature.

(i) If we assume that $M_{i}=M_{i}(0)$ are regular at 0 , then $M$ is covered by a product manifold formed by a flat symmetric space and a symmetric space of compact type.

(ii) The Euler characteristic of $M, \chi(M)$, is defined. If $\chi(M) \neq 0$, then $M$ is isometric to $\mathbf{R}^{k} \times M_{1}$, where $M_{1}$ is a symmetric space of compact type and $k$ is 
the dimension of the subspace of the tangent space of $M$ at the point 0 fixed by the restricted bolonomy group of $M$ at 0 .

Proof. (i) By the same arguments as in Proposition 3, we have the three Riemannian covering maps

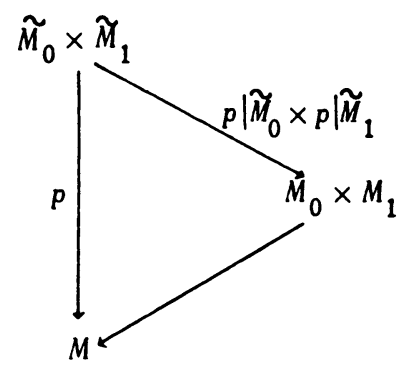

where $M_{0}$ is a flat symmetric space and $M_{1}$ is of compact type.

(ii) The group of deck transformations $\Gamma$ of the covering $p$ lies in $I\left(\tilde{M}_{0}\right) \times$ $I\left(\tilde{M}_{1}\right)$. If $\phi \in \Gamma$, then $\phi=\left(\phi_{0}, \phi_{1}\right)$, where $\phi_{i}: \tilde{M}_{i} \rightarrow \tilde{M}_{i}$ are Clifford translations of $\tilde{M}_{i}$.

By a theorem of Wolf $[10$, p. 271$]$, we see that $\chi(M)$ is well defined and $\pi_{1}(M)$ is finite if $\chi(M) \neq 0$. This means that $\Gamma$ is finite. But a Clifford translation in a Euclidean space is an ordinary translation which is of infinite order. Hence $\underset{\sim}{\phi_{0}}=\mathrm{Id}$, for $\phi=\left(\phi_{0}, \phi_{1}\right) \in \Gamma$. Therefore $M \approx \tilde{M}_{0} \times\left(\tilde{M}_{1 / \mathrm{r}}\right)=\mathbf{R}^{k} \times M_{1}$, where $\widetilde{M}_{0}=\mathbf{R}^{k}$ is the Euclidean factor in $\tilde{M}$. The dimension of $\tilde{M}_{0}$ is exactly the dimension of the subspace of tangent space fixed by the restricted holonomy group of $M$.

We give some further examples.

Example 4. Let $\mathfrak{F}_{1}$ be the foliation in $T^{2}$ with leaves induced from parallel coordinate lines and $\mathfrak{F}_{2}$ the perpendicular foliation. Then $M_{i}(x)=S^{1}$. In this case both foliations are regular and $M_{1}(x) \cap M_{2}(x)=x$. We have a global product $T^{2}=S^{1} \times S^{1}$.

Example 5 (The klein bottle $K$ ). Let $\mathbf{Z} \times \mathbf{Z}$ act on $\mathbf{R} \times \mathbf{R}$ as

$$
(m, n) \cdot(x, y)=(x+m,-y+n) \text {. }
$$

Then $K$ is the orbit space of this action. Let $\left(\mathfrak{F}_{1}, \mathfrak{F}_{2}\right)$ be the local product structure of $K$ induced from the product structure of $\mathbf{R} \times \mathbf{R}$. Denote the projection by $p$ and $(\bar{x}, \bar{y})=$ $p(x, y)$. Then $M_{i}((\bar{x}, \bar{y}))=S^{1}, i=1,2$, for all $(x, y) \in \mathbf{R} \times \mathbf{R}$. At the points $(\bar{x}, \bar{y}), y \neq$ $1 / 2, M_{i}((\bar{x}, \bar{y}))$ are regular at $(\bar{x}, \bar{y}) . \quad M_{1}((\bar{x}, \bar{y})) \times M_{2}((\bar{x}, \bar{y}))=S^{1} \times S^{1}, y \neq 1 / 2$, is a covering space of $K$. At the points $(\bar{x}, \overline{7} / 2), p$ can not factorize through $q_{1} \times q_{2}$ such that $M_{1}((\bar{x}, \overline{1} / 2)) \times M_{2}((\bar{x}, \overline{1} / 2))$ becomes a covering space of $K$, where $q_{i}: \mathbf{R}$ $\rightarrow S^{1}$ is the usual covering map. Neither is $M_{1}((\bar{x}, \pi / 2))$ regular nor does $M_{1}((\bar{x}, \overline{1} / 2))$ satisfy condition (A). In fact, $\tau_{y} X=-X$ for $X$ in $M_{2}((\bar{x}, \bar{T} / 2)), \gamma$ the 
projection of the line segment in $\mathbf{R} \times \mathbf{R}$ joining $(0,1 / 2)$ and $(1,1 / 2)$.

Moreover, $\mathfrak{F}_{2}$ is a regular foliation. For $y \neq 1 / 2$, we have a fibration $S^{1} \rightarrow K$ $\rightarrow S^{1} . K$ is covered by $M_{1}((\bar{x}, \bar{y})) \times M_{2}((\bar{x}, \bar{y}))=S^{1} \times S^{1}$ and the base space $S^{1}$ is covered by $M_{1}((\bar{x}, \bar{y}))=S^{1}$ with a two fold covering. For $y=1 / 2$, we have only the fibration $S^{1}=M_{2}(\bar{x}, \overline{1} / 2) \rightarrow K \rightarrow M_{1}((\bar{x}, \overline{1} / 2))=S^{1}$.

Example $6\left(S^{2} \times S^{3}\right)$. Let $Z_{2}$ act on $S^{2} \times S^{3}$ by $a(x, y)=(-x,-y)$ where $a$ is the nontrivial element of $Z_{2}$. Denote $M=S^{2} \times S^{3} / Z_{2}$. M has two parallel foliations $\mathfrak{F}_{1}$ and $\mathfrak{F}_{2}$ induced from the product structure of $S^{2} \times S^{3}$. For $m \in M$, we have $M_{1}=M_{1}(m)=S^{2}, M_{2}=M_{2}(m)=S^{3}$ and $M$ is covered by $M_{1} \times M_{2}=S^{2} \times S^{3}$. Moreover, we have the fibre bundle $s^{3} \rightarrow M=s^{2} \times s^{3} / \mathbf{Z}_{2} \rightarrow M / \mathfrak{F}_{2}=\mathbf{P}_{2}$ and $\mathbf{P}_{2}$ is covered by $S^{2}$.

Example $7\left(\mathbf{R} \times S^{2}\right)$. Let $\mathbf{Z}$ act on $\mathbf{R} \times S^{2}$ as follows:

$$
2 n \cdot(x, y)=(x+2 n, y), \quad(2 n+1) \cdot(x, y)=(x+2 n+1,-y) .
$$

Denote $p: \mathbf{R} \times S^{2} \rightarrow M=\mathbf{R} \times S^{2} / \mathbf{Z}$. $M$ has an obvious compatible local product structure $\left(\mathfrak{F}_{1}, \mathfrak{F}_{2}\right)$. For $m \in M, M_{1}=M_{1}(m)=S^{1}$ and $M_{2}=M_{2}(m)=S^{2}$, we have the following diagram

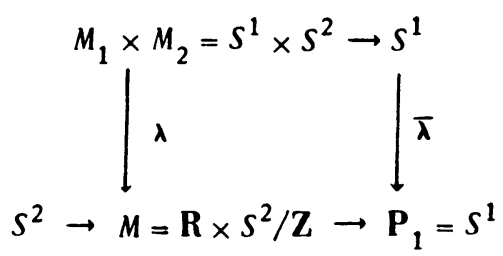

where $\lambda$ and $\bar{\lambda}$ are the 2 -fold coverings.

9. Remarks. One can generalize Theorems 1,2 to affine manifolds and semiRiemannian manifolds. In the affine case, the two complementary, parallel and integrable distributions need not give a local affine product structure. One needs the additional assumption that the manifold is locally an affine local product of two neighborhoods of the two leaves (see [2], [11], [12]). In the semi-Riemannian case, one assumes that the metric restricted to $T_{m}^{1}$ is nondegenerate to get the semi-Riemannian local product structure [11]. As for the proof, one replaces the notions in Riemannian manifolds by the corresponding notions in semi-Riemannian manifolds and affine manifolds. For example, replace complete Riemannian manifold by complete affine manifold (geodesic completeness); isometry group by affine group; Riemannian covering map by affine covering map and so forth. Then everything works except Lemma 2. To prove Lemma 2 in the semi-Riemannian and the affine cases one uses the theorem of $\mathrm{H}$. Wu [11] and the theorem of Kashiwabara [2] instead of the theorem of de Rham. To prove Lemma 7 in the affine case one has to reparametrize each geodesic in a corresponding normal flat 
neighborhood by affine parameters. One obtains a unique parameter $t$ for every geodesic starting at $\gamma$ and a number $t_{0}$ such that $\exp t X$ and $\left.\exp t \sigma_{\gamma} X\right)$ lie in the same leaf for $0 \leq t \leq t_{0}$. From this one proves Lemma 7 using the regularity. This generalizes Theorems 1,2 to semi-Riemannian manifolds and affine manifolds.

\section{BIB LIOGRA PHY}

1. C. Chevalley, Theory of Lie groups. I, Princeton Math. Series, vol. 8, Princeton Univ. Press, Princeton, N. J., 1946. MR 7, 412.

2. S. Kashiwabara, On the reducibility of an affinely connected manifold, Tôhoku Math. J. (2) 8 (1956), 13-28. MR 18, 332.

3. S. Kobayashi and K. Nomizu, Foundations of differential geometry. I, Interscience, New York, (1963). MR 27 \#2945.

4. R.S. Palais, A global formulation of the Lie theory of transformation groups, Mem. Amer. Math. Soc. No. 22 (1957). MR 22 \#12162.

5. G. Reeb, Sur certaines propriétés topologiques des variétés feuilletées, Actualités Sci. Indust., no. 1183 = Publ. Inst. Math. Univ. Strasbourg 11, Hermann, Paris, 1952, pp. 91-154, 157-158. MR 14, 1113.

6. G. de Rham, Sur la réductibilité d'un espace de Riemann. Comment. Math. Helv. 26 (1952), 328-344. MR 14, 584.

7. B. Reinhart, Foliated manifolds with bundle-like metrics, Ann. of Math. (2) 69 (1959), 119-132. MR $21 \# 6004$.

8. S. A. Robertson, Grid manifolds, J. Differential Geometry 4 (1970), 245-253. MR 42 \#3711.

9. J. A. Wolf, Locally symmetric homogeneous spaces, Comment. Math. Helv. 37 (1962/63), fasc. 1, 65-101. MR 26 \#5522.

10. - On locally symmetric spaces of non-negative curvature and certain other locally homogeneous spaces, Comment. Math. Helv. 37 (1962/63), fasc. 4, 266-295. MR 27 \#4178.

11. H. Wu, On the de Rham decomposition theorem, Illinois J. Math. 8 (1964), 291311. MR $28 \# 4488$.

12. - Decomposition of Riemannian manifolds, Bull. Amer. Math. Soc. 70 (1964), 610-617. MR 29\#566.

DEPARTMENT OF MATHEMATICS, UNIVERSITY OF ILLINOIS AT URBANA-CHAMPAIGN, URBANA, ILLINOIS 61801

Current address: Department of Mathematics, University of Notre Dame, Notre Dame, Indiana 46556 